\title{
Política en los sitios web, entretenimiento en las redes: la agenda de los medios noticiosos en sus sitios y en Facebook y Twitter
}

\section{Politics on the homepage, entertainment on social media: news media agenda on their homepages, Facebook and Twitter}

\author{
Panza Guardatti, M., Mitchelstein, E. y Boczkowski, P. J. ${ }^{1}$ \\ Recibido: 20-09-2018 - Aceptado: 27-01-2019 \\ DOI: https://doi.org/10.26441/RC18.1-2019-A7
}

\begin{abstract}
RESUMEN: Esta investigación examina la agenda online de dos de los diarios más relevantes en Argentina -Clarín y La Nación- a través de un análisis cuantitativo de las noticias publicadas en las respectivas páginas de inicio y en las respectivas publicaciones en las cuentas oficiales de estos dos medios en Facebook y Twitter. El análisis de 3.780 noticias demuestra que existen diferencias entre las agendas propuestas por los sitios y las noticias publicadas en las redes sociales de los mismos. Los resultados exponen que los diarios mantienen una agenda relacionada a los asuntos públicos en sus páginas de inicio, mientras que en las redes sociales priorizan noticias vinculadas a los asuntos no públicos. La divergencia es menor temprano por la mañana y aumenta a lo largo del día. A partir de estos hallazgos este trabajo indaga los motivos de esta selección y las diferentes temáticas que seleccionan los medios.
\end{abstract}

Palabras clave: agenda de noticias; redes sociales; periodismo; noticias en línea; ciclo de noticias.

ABSTRACT: This work examines the online agenda of two of the most important newspapers in Argentina -Clarín and La Nación- through quantitative content analysis of the news stories published on their respective homepages and on the official accounts of these two media outlets on Facebook and Twitter. The analysis of 3.780 news shows that newspapers maintain an agenda related to public affairs on their home pages, while on social networks they prioritize news related to non-public topics. The divergence is lowest early in the morning and increases throughout the day. This paper explores the reasons for the differences between the topics selected by the news companies on their homepages and their social media presence.

Keywords: news agenda; social media; journalism; news online; news cycle.

\footnotetext{
Mattía Panza Guardatti es Licenciado en Comunicación por la Universidad de San Andrés (Argentina). mpanzaguardatti@udesa.edu.ar. https://orcid.org/0000-0003-0726-9013

Eugenia Mitchelstein es Doctora del Departamento de Comunicación de Northwestern University, Profesora adjunta en la Universidad San Andrés y y co-directora del Centro de Estudios sobre Medios y Sociedad en Argentina.. emitchelstein@udesa.edu.ar, https://orcid.org/0000-0001-7355-8740

Pablo J. Boczkowski es Ph.D. en Science and Technology Studies (Cornell University), profesor en el Department of Communication Studies en Northwestern University y co-director del Centro de Estudios sobre Medios y Sociedad en Argentina.. pjb9@northwestern.edu. https://orcid.org/0000-0002-9446-8303
} 


\section{Introducción}

La conformación de la agenda mediática ha sido tema de investigación para los estudios sobre comunicación desde el trabajo seminal de McCombs (1972). El surgimiento de las redes sociales y su utilización como espacios de consumo de información han ampliado el foco de análisis hacia las cuentas en redes sociales de medios de noticias (Conway, Kenski y Wang, 2015; Johnson y Perlmutter, 2009; Martin y Grüb, 2016; Meraz, 2009; Sayre, Bode, Shah, Wilcox, y Shah, 2010). Este trabajo examina la agenda online de los dos diarios impresos con mayor circulación en Argentina -Clarín y La Nación- comparando las noticias publicadas en tres presentaciones de los dos medios: sus páginas de inicio y las publicaciones en las cuentas oficiales de estos dos medios en Facebook y Twitter, durante 55 días no consecutivos en 2017 y 2018.

El análisis muestra que Clarín y $L a$ Nación tienden a publicar más artículos sobre asuntos no públicos en las redes sociales. Mientras que en las páginas de inicio los asuntos públicos ocupan un porcentaje promedio de $56,43 \%$, en Twitter las noticias de asuntos públicos disminuyen en promedio a $37,15 \%$, y en Facebook llegan a una media de $34,05 \%$ de los posteos. Además, aunque la brecha entre el porcentaje de asuntos públicos en las páginas de inicio y las redes es una constante en todos los horarios, hay mayor proporción de noticias sobre asuntos públicos a primera hora de la mañana en todos los espacios de difusión de los dos diarios, que disminuye hacia a la tarde y la noche. Este trabajo reflexiona sobre la fijación de agenda online y en redes sociales a partir de estos hallazgos, con tres conclusiones principales: la lógica de las redes sociales incide en la difusión de noticias por parte de actores establecidos de la industria; las prácticas en cada red social difieren, y los medios de noticias se adaptan a esos usos diversos, proponiendo agendas distintas para cada espacio virtual; y la persistencia de los asuntos de temas en las redes en las primeras horas de la mañana sugiere que los diarios mantienen su relevancia a la hora de fijar agenda al principio del día.

\section{Marco Referencial}

\subsection{Agenda setting.}

En 1963, Cohen propuso que "la prensa puede no tener éxito en decirle a la gente cómo tiene que pensar, pero ha sido sorprendentemente exitosa en decirle a sus lectores sobre qué temas pensar"2 (p.13). McCombs y Shaw retoman ese concepto y definen a la fijación de agenda como "la capacidad de los medios masivos de seleccionar y destacar ciertos temas sobre otros, y con ello causar que los asuntos destacados sean percibidos como importantes por el público" (1977, p.12). El proceso de fijación de agenda está formado por tres elementos interrelacionados: la agenda de

2 Todas las traducciones son de los autores. 
los medios, la agenda pública y la agenda política o institucional (Chaffee y Metzger, 2001; Dearing y Rogers, 1996; Rodríguez Díaz, 2004; Aruguete, 2009). El primer nivel de la agenda, llamado también "agenda de los medios", está definido por la jerarquía y frecuencia otorgadas a distintos temas en los medios de comunicación (Aruguete, 2009).

La "agenda del público" es el grado de importancia que da el público a determinados aspectos noticiosos durante un periodo de tiempo (Dearing y Rogers, 1996) y se relaciona con la medición de la importancia que tiene la selección de determinados temas entre la audiencia (McCombs y Evatt, 1995). El tercer nivel de agenda, política e institucional, se refiere a la relación entre la agenda pública y las decisiones y acciones de las elites y los tomadores de decisiones (Dearing y Rogers, 1988). La investigación académica se ha concentrado en la influencia que tienen los medios en la opinión pública (López Escobar, Llamas y McCombs, 1996; Weaver y Elliott, 1985; Mc Combs y Evatt; 1995; Semetko y Canel, 1997; Kosicki, 1993).

Algunas investigaciones examinan cómo el surgimiento de Internet puso en cuestión el potencial de los medios de noticias tradicionales para fijar la agenda del público (Althaus y Tewksbury, 2002; Boczkowski y Mitchelstein, 2013; Chaffee y Metzger, 2001; Donsbach, 2004; Roberts, Wanta y Zwo, 2002). McCombs vaticinó el final del proceso de fijación de agenda:

Internet ha cambiado dramáticamente el espacio comunicacional con la introducción de una miríada de canales (...) hay observadores que predicen el final de la fijación de agenda conforme las audiencias se fragmentan y prácticamente todo el mundo tiene una agenda externa única, altamente individualizada y compuesta por la gran cantidad de información disponible online (2005; p.544).

Los primeros estudios sobre fijación de agenda online examinan la interacción entre las agendas de los medios tradicionales y los nuevos medios, como los blogs (Sweetser, Golan y Wanta 2008; Wright y Hinson, 2008; Messner y Garrison, 2011; Messner y DiStaso, 2008). La aparición de las redes sociales plantea un nuevo interrogante sobre los efectos de la fijación de agenda de los medios. La literatura se ha enfocado en analizar la influencia de los medios tradicionales de noticias en redes sociales como Twitter y Facebook (Campos Freire 2008; Meraz, 2011; Lara, 2008; Noguera Vivo, 2010; Sayre et al., 2010). Por ejemplo, Harder y sus colegas encuentran que las cuentas de medios en Twitter tienen mayor poder de fijación de agenda que otro tipo de cuentas (Harder, Sevenans y Van Aelst, 2017). Kalsnes examina la fijación de agenda entre Twitter y seis diarios noruegos, y encuentra que las referencias a tweets de representantes políticos aumentan conforme se acerca la fecha de la elección (2016). En cambio, otra investigación encuentra que, durante la campaña electoral de 2012 en Estados Unidos, tanto los medios como los candidatos influyeron en la agenda de Twitter (Conway, Kenski y Wang, 2015). 


\subsection{Noticias y redes sociales.}

Las redes sociales aparecen como nuevas plataformas para la producción y consumo de información (Gil De Zuñiga, Jung y Valenzuela 2012; Hong, 2012; Mitchelstein y Boczkowski, 2018; Newman, Fletcher, Kalogeropoulos, Levy y Nielsen, 2017). La investigación ha dado cuenta de cómo los medios de noticias usan estrategias para posicionarse en estos nuevos espacios (García De Torres, et al., 2011; González Molina y Ortells Badenes; 2012; Hille y Bakker, 2013; Hong 2012). Sin embargo, Ju, Jeong y Chyi examinan la circulación, el tráfico online y a las audiencias de 66 diarios impresos de los Estados Unidos y encuentran que "el rol de los medios sociales para el modelo de negocios de los periódicos (es decir, su contribución al tráfico web y a sus ingresos totales) sigue estando indefinido en el mejor de los casos" (2014, p. 13).

Cada red social tiene lógicas diferentes de funcionamiento e interacción para los usuarios (Boczkowski, Matassi y Mitchelstein, 2018), incluyendo los medios que las usan para difundir su contenido. Estas diferencias reflejan en parte la diversidad de audiencias: los usuarios de Twitter tienden a ser más jóvenes, y hay más mujeres que hombres en Facebook (Kümpel, Karnowski y Keyling, 2015).

Las investigaciones sobre Twitter indican que es un espacio para la "expresión del discurso dominante en la opinión pública, pero también como un instrumento del sistema político para dar forma a la cobertura noticiosa" (Aruguete, 2015, p. 170). Su usabilidad, una vasta cantidad de usuarios, presencia de personajes públicos y sobre todo su instantaneidad la hacen útil para la difusión de noticias (Boyd, Golder, Lotan, 2010; Kwak, Lee, Park y Moon; 2010). Algunos trabajos comparan la agenda noticiosa de los medios con los temas que se presentan en Twitter y encuentran que existe gran influencia de esta red social sobre la agenda noticiosa (Parmelee, 2014; Krane, 2010). En esta plataforma, los periodistas aparecen como formadores de opinión y tratan los temas desde sus cuentas personales (Lasorsa, Lewis y Holton, 2012; Vis, 2013) Aruguete propone que Twitter es capaz de competir por el poder de agenda de los medios tradicionales (2015).

Si bien la mayor parte de los estudios se enfocan en Twitter, debido a que es una red abierta, "en la que es mucho más fácil seguir usuarios clave, desarrollos temporales, y redes de comunicación” (Kümpel, Karnowski y Keyling, 2015, pp. 3-4 ), la investigación académica sobre la fijación de agenda por parte de Facebook (Johnson y Perlmutter, 2009; Martin y Grüb, 2016; Jacobson, 2013) se ha concentrado en los usos que medios y periodistas hacen de esta red (González Molina y Ramos del Cano; 2013; González Molina y Ortells Badenes; 2012). Los estudios indican que los medios utilizan a esta red social como vidriera, es decir para difundir la información producida y redirigir a los usuarios hacia el sitio nativo (Canavilhas, 2007; Jerónimo y Duarte, 2010; Jung y Villi, 2018; Noguera Vivo, 2010; Skogerb $\varnothing$ y Krumsvik, 2015). 
Por parte de los usuarios, varios trabajos indican que los consumidores utilizan las redes sociales para acceder a noticias (Mitchelstein y Boczkowski, 2018; Newman, Fletcher, Kalogeropoulos, Levy y Nielsen, 2017). Por ejemplo, Boczkowski, Mitchelstein y Matassi encuentran que los jóvenes en Argentina adquieren información en redes sociales de manera tanto deliberada como incidental (2018). La agenda propuesta por las redes sociales y la agenda de sus usuarios no necesariamente está centrada en la información sobre asuntos públicos. Estudios sobre el consumo de contenido en redes encuentran que gran parte de los intercambios de información en las redes no está relacionada con asuntos noticiosos (Boczkowski, Matassi y Mitchelstein, 2018; Fletcher y Nielsen, 2017; Oeldorf Hirsch, Birnholtz y Hancock, 2017; Valeriani y Vaccari, 2016). Bandari, Asur y Huberman (2012) concluyen que los contenidos publicados por los medios tradicionales de noticias y relacionados a los asuntos públicos no suelen ser los más populares en Twitter.

Los trabajos sobre redes sociales en Argentina encuentran alto grado de correspondencia entre el contenido de los sitios y los posteos en sus cuentas de redes sociales. Raimondo Anselmino y Bertone resaltan que "tanto en la cuenta oficial de Clarín como en la de La Nación, encontramos correspondencia entre las noticias emitidas desde ese espacio [Twitter] y las que se ubican en la portada (home) del sitio." (2013, p. 97). Por otra parte, Rost (2012) indica que en las redes sociales se produce el "volcado automático. Es la modalidad más usual en los medios. Se utiliza alguna aplicación de distribución de RSS (Really Simple Sindication) y se vuelcan automáticamente las últimas noticias que se publican en la Web en cada una de las redes sociales" (p. 5).

Sin embargo, la similitud entre las agendas mediáticas puede variar de acuerdo al momento del día, como encuentran Boczkowski y De Santos en su comparación entre las páginas de inicio de Clarín, La Nación e Infobae (2007). Las variaciones temporales en el tipo y cantidad de información publicada se deben a los cambios en el ciclo de las noticias, que es la organización de la difusión de contenido a lo largo del día. La investigación empírica ha dado cuenta de cómo la aceleración en el trabajo periodístico online y en los canales de cable de noticias ha contribuido al fin de la práctica de difundir información dos veces por día (a la mañana y a la noche) (Boczkowski, 2009; Lawson Borders, 2006; Williams y Delli Carpini, 2000). Esta organización temporal es remplazada por un ciclo de noticias de 24 horas, en el que se produce y distribuye información de manera constante (Bucy, Gantz y Wang, 2007). Klinenberg hace un estudio etnográfico del trabajo periodístico y propone que "el ciclo de tiempo para la producción de noticias en la era de la producción digital es radicalmente diferente: el ciclo de noticias regulares se ha convertido en un patrón errático e interminable (...) un ciclón de noticias" (2005, p. 54).

Aunque algunos autores han examinado la interacción entre el ciclo de noticias y las redes sociales, (Castillo, El 
Haddad, Pfeffer y Stempeck, 2014; Kalsnes, 2016; Lescovek, Backstrom y Kleinberg, 2009; Sayre et al., 2010), la mayoría de las investigaciones sobre las agendas de medios y redes sociales recolecta los datos durante un lapso breve de tiempo en un solo momento al día, o compara las cuentas de medios de noticias en redes sociales con cuentas de otros usuarios. Este trabajo de investigación compara las páginas de inicio y las cuentas de Twitter y Facebook de los dos diarios de mayor circulación en la Argentina entre los meses de febrero de 2017 y marzo de 2018. Además, siguiendo la metodología propuesta por Boczkowski y De Santos (2007), examina si existen diferencias en tres horarios distintos del día. De esta manera, analiza las variaciones de las agendas intra-medio pero inter-red social. La existencia de diferencias entre páginas de inicio de La Nación y Clarín y sus cuentas en redes sociales indicaría que incluso dentro de cada medio noticioso, el criterio editorial para construir la agenda mediática varía de acuerdo al espacio de publicación.

Pregunta de Investigación e Hipótesis

Este trabajo propone dos hipótesis y una pregunta de investigación:

H1: Los medios publican más noticias de asuntos públicos en sus páginas de inicio que en sus cuentas de Facebook.

H2: Los medios publican más noticias de asuntos públicos en sus páginas de inicio que en sus cuentas de Twitter.

P.I.: ¿Varían los temas de la agenda mediática a lo largo del día en los tres espacios?

\section{Metodología}

Esta investigación examina la agenda periodística en dos sitios de noticias online y en las redes Facebook y Twitter. Para llevarla a acabo, replica la metodología utilizada en Boczkowski y Mitchelstein (2015). Realiza un análisis de contenido cuantitativo (Krippendorff, 2018; Kripendorff y Bock, 2008) de las notas publicadas en Clarin (www.clarin. com.ar) y La Nación (www.lanacion.com. ar) y en sus respectivas cuentas de Facebook (@clarin y @lanacion) y Twitter(@clarincom; @lanacion).

Los datos de la primera muestra se recolectaron en 48 días durante los meses de febrero, abril, junio, agosto, noviembre y diciembre del año 2017 a partir del método de la semana construida, e incluyen un total de 2.520 noticias. La semana construida consiste en elegir el lunes de la primera semana del período designado, luego el martes de la segunda semana y así consecutivamente hasta cumplir una semana calendario (Krippendorf, 2018). Este modo de selección permite obtener una muestra representativa de la información y establece un rango de selección relativamente considerable para la conformación de un corpus de análisis de contenido. El muestreo mediante semana construida evita que acontecimientos puntuales como una campaña presidencial o una competencia deportiva sesgue los resultados del análisis.

A su vez, se elaboró una segunda muestra, recolectando los datos día de por medio durante dos semanas (7 días en total), con el examinar si había modificaciones en la agenda mediática de 
acuerdo al momento del día. De esta manera, durante el mes de marzo de 2018, se recolectaron 1.260 noticias, de los mismos sitios, en distintos horarios del día (08:00, 16:00 y 00:00).

Este estudio se llevó a cabo en dos de los diarios más representativos de la Argentina, por diversas razones. Clarín y La Nación están ponderados por diversos rankings como dos de los sitios más populares en ese país. ${ }^{2}$ Este trabajo analiza las cuentas de estos diarios en Facebook y Twitter porque son dos de las redes sociales con más usuarios de Argentina. ${ }^{3}$ Tanto Clarín como La Nación ocupan un lugar de relevancia en las redes sociales. Cuentan con caudales elevados de posteos y sobre todo de seguidores. En Twitter, Clarín(@ clarincom) tiene un total de 2.79 millones de seguidores en su cuenta verificada y $L a$ Nación (@LANACION) tiene 3.13 millones de seguidores (julio 2018). En Facebook, la página de Clarín (@clarincom) tiene 5.717.723 seguidores, y la de La Nación (@lanacion) tiene 3.564.287 seguidores (julio 2018).

La agenda de los sitios se midió en función de las 10 noticias que aparecen primero en la pantalla de las páginas de inicio de los diarios online (en una grilla de izquierda a derecha y de arriba abajo). Se utilizó este criterio de recolección entendiendo que aquellas noticias que aparecen en la parte superior del sitio son las consideradas más "importantes" por los editores $y$, por ende, son un indi- cador de lo más relevante para los productores de noticias (Boczkowski y Mitchelstein, 2015). En el caso de la agenda de las redes sociales, se recolectaron las 10 noticias que aparecen primeras en la pantalla de inicio de las respectivas cuentas de los medios.

Las unidades de análisis de este estudio son las notas y las publicaciones en redes sociales. Las variables codificadas fueron las siguientes:

- Lista:

- Página de inicio.

- Facebook.

- Twitter.

- Contenido:

- Asuntos públicos (AP): una noticia fue categorizada como de asuntos públicos si involucraba temas de economía, de gobierno, de política, de negocios, relaciones internacionales, o si involucraba a figuras públicas o tenía una implicancia política explícita.

- Asuntos no públicos (ANP): noticias sobre deportes, entretenimiento, crimen, tecnología, cultura, educación, el clima o toda noticia que no tuviera ningún tipo de vinculación con los asuntos públicos.

La primera variable responde a los contenidos publicados en la página de inicio de los sitios y las noticias publicadas en las páginas de Facebook y Twitter. La segunda variable investiga la diferencia entre las preferencias que tienen los editores a la hora de seleccionar qué no-

\footnotetext{
2 En marzo de 2018, Clarín tuvo 5.397.000 usuarios únicos, y La Nación 4.902.000 usuarios únicos (Comscore, 2018).

3 En marzo de 2018 Facebook tuvo 17.061 millones de usuarios únicos; y Twitter 3.333 millones de usuarios únicos (Comscore, 2018)
} 
ticias serán publicadas en el sitio, en las redes o en ambas. La categorización de ambas variables está basada en el libro de Boczkowski y Mitchelstein (2015). La fiabilidad inter-codificadores fue en promedio $85 \%$ con un coeficiente de Cohen Kappa de 0.81.

\section{Hallazgos}

\subsection{Composición de la muestra.}

Aunque las agendas de los dos medios examinados divergen, existe una convergencia en las temáticas de las noticias según se publique en los sitios o las redes sociales. En las páginas de inicio de $L a$ Nación y Clarín hay más noticias sobre asuntos públicos que noticias sobre asuntos no públicos. La Tabla 1 muestra porcentaje de noticias de asuntos públicos (AP) y asuntos no públicos (ANP) en las páginas de inicio y las cuentas de Facebook y Twitter de los diarios Clarín y La Nación, recolectadas a las 18:00 en 48 días de los meses de febrero, junio, agosto, noviembre y diciembre de 2017. La prevalencia de las noticias sobre asuntos públicos en ambas páginas de inicio es $50.24 \%$ y $62.62 \%$ respectivamente. En la página de Facebook de Clarín hay 28,81\% de noticias sobre asuntos públicos y
$71.19 \%$ asuntos no públicos. Esto significa que hay una brecha significativa en el porcentaje de noticias sobre asuntos públicos de 21 puntos porcentuales entre la página de inicio de Clarín y su página de Facebook. En la página de Facebook de La Nación hay $39,29 \%$ de noticias sobre asuntos públicos y $60,71 \%$ de asuntos no públicos. La brecha en el porcentaje de noticias sobre asuntos públicos entre la página de inicio y Facebook es de significativa y de 23 puntos porcentuales. Estos hallazgos confirman la $\mathrm{H} 1$ para los dos medios estudiados.

En la cuenta de Twitter de Clarín las noticias sobre asuntos no públicos $(58,33 \%)$ superan a las de asuntos públi$\cos (41,67 \%)$. La diferencia en el porcentaje de noticias sobre asuntos públicos entre el sitio de Clarín y su cuenta de Twitter es significativa y de 9 puntos porcentuales. En la cuenta de Twitter de La Nación el porcentaje de notas sobre asuntos no públicos $(67,38 \%)$ también supera el porcentaje de artículos sobre asuntos públicos (32.62\%). La brecha en el porcentaje de noticias sobre asuntos públicos entre el sitio de La Nación y su cuenta de Twitter es de 30 puntos porcentuales. Estos hallazgos confirman la H2 para los dos medios examinados.

Tabla 1. Porcentaje de noticias de asuntos públicos (AP) y asuntos no públicos (ANP) de los diarios Clarín y La Nación

\begin{tabular}{|l|l|l|l|l|l|l|}
\hline & \multicolumn{3}{|c|}{ Clarín } & \multicolumn{2}{l|}{ La Nación } \\
\hline & & AP & ANP & & AP & ANP \\
\hline Homepage & $\mathrm{N}=420$ & $50,24 \%$ & $49,76 \%$ & $\mathrm{~N}=420$ & $62,62 \%$ & $37,38 \%$ \\
\hline Twitter & $\mathrm{N}=420$ & $41,67 \%$ & $58,33 \%$ & $\mathrm{~N}=420$ & $32,62 \%$ & $67,38 \%$ \\
\hline Facebook & $\mathrm{N}=420$ & $28,81 \%$ & $71,19 \%$ & $\mathrm{~N}=420$ & $39,29 \%$ & $60,71 \%$ \\
\hline
\end{tabular}

Fuente: Elaboración en propia sobre la base de datos recolectados para esta investigación. 
Significancia estadística de la diferencia entre el porcentaje de noticias de asuntos públicos en la página de inicio y

Clarin Homepage vs. Facebook:

Clarin Homepage vs. Twitter:

Clarín Facebook vs. Twitter:

La Nación Homepage vs. Facebook:

La Nación Homepage vs. Twitter

La Nación Facebook vs. Twitter:

Para examinar si el porcentaje de noticias sobre asuntos públicos varía a lo largo del día (P.I.), analizamos los datos recolectados en tres horarios diferentes (a las 08:00, 16:00 y 0:00). El mayor porcentaje de noticias de asuntos públicos aparece en la página de inicio de cada sitio a las 8:00 (53\% en Clarín y 54\% en La Nación). La Tabla 2 el porcentaje de noticias sobre asuntos públicos en las redes sociales de Clarín y La Nación:

$$
\begin{aligned}
& X^{2}=40,3425 \text { Pr }<0,0005 \\
& X^{2}=6,2121 \quad \operatorname{Pr}=0,013 \\
& X^{2}=15,2116 \operatorname{Pr}<0,0005 \\
& X^{2}=45,7499 \operatorname{Pr}<0,0005 \\
& X^{2}=75,7718 \text { Pr }<0,0005 \\
& X^{2}=15,2116 \operatorname{Pr}<0,0005
\end{aligned}
$$

visualiza porcentaje de noticias sobre asuntos públicos en las páginas de inicio y las cuentas de Facebook y Twitter de los diarios Clarín y La Nación, recolectadas a las 8:00, 16:00 y 0:00, durante 7 días entre el 19 de marzo y el 31 del mismo mes. A las 16:00, los porcentajes son $47 \%$ y $53 \%$, y a las 0:00 son $46 \%$ y $49 \%$, respectivamente.

Tabla 2. Porcentaje de noticias sobre asuntos públicos de los diarios Clarín y La Nación, recolectadas a las 8:00, 16:00 y 0:00

\begin{tabular}{|l|l|l|l|l|l|l|l|l|}
\hline & \multicolumn{9}{|l|}{ Clarín } & \multicolumn{3}{l|}{ La Nación } \\
\hline & $\mathbf{8 : 0 0}$ & $\mathbf{1 6 : 0 0}$ & $\mathbf{0 : 0 0}$ & Total & $\mathbf{8 : 0 0}$ & $\mathbf{1 6 : 0 0}$ & $\mathbf{0 : 0 0}$ & Total \\
\cline { 2 - 9 } & $\mathbf{N = 2 1 0}$ & $\mathbf{N}=\mathbf{2 1 0}$ & $\mathbf{N}=\mathbf{2 1 0}$ & $\mathbf{N}=\mathbf{6 3 0}$ & $\mathbf{N}=\mathbf{2 1 0}$ & $\mathbf{N}=\mathbf{2 1 0}$ & $\mathbf{N}=\mathbf{2 1 0}$ & $\mathbf{N}=\mathbf{6 3 0}$ \\
\hline Página de inicio & $52.86 \%$ & $47.14 \%$ & $45.71 \%$ & $50,24 \%$ & $54.29 \%$ & $52.86 \%$ & $48.57 \%$ & $51,90 \%$ \\
\hline Facebook & $55.71 \%$ & $11.43 \%$ & $34.29 \%$ & $33,81 \%$ & $48,57 \%$ & $21,43 \%$ & $25,71 \%$ & $31,90 \%$ \\
\hline Twitter & $47.14 \%$ & $32.86 \%$ & $45.71 \%$ & $41,90 \%$ & $31.43 \%$ & $32,86 \%$ & $25,71 \%$ & $30 \%$ \\
\hline
\end{tabular}

Fuente: Elaboración en propia sobre la base de datos recolectados para esta investigación.

Significancia estadística de la diferencia entre el porcentaje de noticias de asuntos públicos en la página de inicio y

Clarin 0:00: Homepage vs. Facebook:

Clarin 0:00: Homepage vs. Twitter:

Clarín 0:00: Facebook vs. Twitter:

Clarin 8:00: Homepage vs. Facebook:

Clarin 8:00: Homepage vs. Twitter: el porcentaje de noticias sobre asuntos públicos en las redes sociales de Clarín y La Nación.

$$
\begin{array}{ll}
X^{2}=1,9048, & \text { Pr }=0,168 \\
X^{2}=0,0000 & \text { Pr }=1,000 \\
X^{2}=1,9048 & \text { Pr }=0,168 \\
X^{2}=0,1151 & \text { Pr }=0,734 \\
X^{2}=0,4571 & \text { Pr }=0,499
\end{array}
$$


Clarin 8:00: Facebook vs. Twitter:

Clarín 16:00: Homepage vs. Facebook:

Clarín 16:00: Homepage vs. Twitter:

Clarin 16:00: Facebook vs. Twitter:

La Nación 0:00: Homepage vs. Facebook:

La Nación 0:00: Homepage vs. Twitter:

La Nación 0:00: Facebook vs. Twitter:

La Nación 8:00: Homepage vs. Facebook:

La Nación 8:00: Homepage vs. Twitter:

La Nación 8:00: Facebook vs. Twitter:

La Nación 16:00: Homepage vs. Facebook:

La Nación 16:00: Homepage vs. Twitter:

La Nación 16:00: Facebook vs. Twitter:

En Facebook, la primera hora de la mañana también es el horario de mayor presencia de contenido sobre asuntos públicos (56\% en Clarín y $49 \%$ en $\mathrm{La}$ Nación), que disminuye a las 16:00 a 11\% y $21 \%$, respectivamente; y aumenta a $34 \%$ en Clarín y a $25 \%$ en La Nación a las 0:00, pero sin llegar al nivel de las 8:00.

En Twitter, a las 8:00 hay 47\% de tweets sobre asuntos públicos en Clarín y $31 \%$ en La Nación. A las 16:00 disminuye a $33 \%$ en Clarín y se mantiene relativamente estable en $33 \%$ en $\mathrm{La}$ Nación, y a las 0:00 es de 46\% en Clarín y $26 \%$ en La Nación. Estos hallazgos indican que el momento de mayor presencia de noticias de asuntos públicos tanto en los sitios de los diarios como en sus cuentas de redes sociales $-y$ por lo tanto, la instancia en que mayor coincidencia hay entre las agendas de las páginas de inicio y las de las cuentas de Facebook y Twitter- es a primera hora la mañana. En Clarín, tanto en la página de inicio como en las dos redes, hay más temas asuntos no públicos a las 16:00, y los mismos disminuyen a me-

$$
\begin{array}{ll}
X^{2}=1,0294 & \operatorname{Pr}=0,310 \\
X^{2}=221,5570 & \operatorname{Pr}<0,0005 \\
X^{2}=2,9762 & \operatorname{Pr}=0,084 \\
X^{2}=9.3223 & \operatorname{Pr}=0.002 \\
X^{2}=7,8322 & \operatorname{Pr}=0,005 \\
X^{2}=7,8322 & \operatorname{Pr}=0,005 \\
X^{2}=0,0000 & \operatorname{Pr}=1,000 \\
X^{2}=0,4575 & \operatorname{Pr}=0,499 \\
X^{2}=7,4667 & \operatorname{Pr}=0,006 \\
X^{2}=4,2857 & \operatorname{Pr}=0,038 \\
X^{2}=14,8077 & \operatorname{Pr}<0.0005 \\
X^{2}=5,7167 & \operatorname{Pr}=0,017 \\
X^{2}=2,3117 & \operatorname{Pr}=0,128
\end{array}
$$

dianoche. En cambio, en La Nación, los asuntos no públicos van ganando espacio conforme avanza el día, para llegar a su máximo a las 0:00.

\section{2. Discusión}

Los hallazgos presentados en este trabajo indican que Clarín y La Nación publican más noticias sobre asuntos públicos en el espacio más visible de sus páginas de inicio que en sus cuentas en las redes sociales Facebook y Twitter, donde publican más artículos relacionados a los asuntos no públicos. En análisis en distintos momentos del día indica que la agenda tanto de las páginas de inicio como de las cuentas en redes sociales incluye más temas de asuntos públicos a las 8 de la mañana que durante el resto el día.

La variación entre las páginas de inicio y las redes indica que los medios analizados abandonaron la práctica de replicar todo su contenido en los lugares más prominentes en sus cuentas de Facebook y Twitter, como estudios anteriores habían encontrado (Rost, 2012; 
Bertone y Raimondo Anselmino, 2013). El hecho de que las noticias de asuntos no públicos ocupen más espacio en Facebook y Twitter, en ese orden, que en las páginas de inicio, parece indicar que en estas plataformas los medios se acercan más a las preferencias del público, que se orienta más por esta temática que hacia las noticias de asuntos públicos (Boczkowski y Mitchelstein, 2013; Tewksbury, 2003; Prior, 2007).

El aumento de noticias sobre asuntos no públicos tanto en las páginas de inicio como en Facebook y Twitter reproduce lo hallado por Boczkowski y de Santos (2007) respecto a la evolución temporal del ciclo informativo, y, como estos autores proponen, desafía la idea de la desaparición del ciclo de noticias o su reemplazo por un ciclón de noticias (Klinenberg, 2005). Las 8 de la mañana es el horario en que la mayoría de los lectores de la versión impresa de Clarín y La Nación se encuentran con la tapa del diario en papel, que tiende a estar dominada por temas de asuntos públicos (Boczkowski y de Santos, 2007). Esta preponderancia de temas de asuntos públicos se repite en Facebook y Twitter, lo que indicaría la continuidad de prácticas editoriales del diario impreso en las plataformas digitales.

$\mathrm{Si}$, como proponen Chaffee y Metzger, "el problema clave para la teoría de la fijación de la agenda pasará de examinar sobre qué temas les dicen los medios a la gente que deberían pensar, a estudiar cómo las audiencias les dicen a los medios sobre qué quieren pensar" (2001, p. 375), las redes sociales se convierten una pieza clave para la transmi- sión de los intereses de las audiencias, tanto para las organizaciones de noticias como para los especialistas en comunicación. Los resultados coinciden con los estudios que señalan que Twitter es una red social en donde se discuten temas de la vida pública (Aruguete, 2015; Boczkowski et al., 2018; Vis, 2013), mientras que las cuentas de los medios en Facebook tienden a privilegiar aún más temas sobre asuntos no públicos (Boczkowski et al., 2018). Facebook anunció públicamente en enero de 2018 que cambiaría su algoritmo para que privilegiara posteos de amigos o familia y organizaciones locales, que tienden a ser sobre asuntos no públicos (Chaykowski, 2018) por encima de noticias publicadas por medios de noticias nacionales. La relativa escasez de noticias sobre asuntos públicos en las cuentas de Facebook de medios tradicionales podría estar relacionada con una estrategia para maximizar la audiencia.

Este trabajo presenta algunas limitaciones. Primero, únicamente analiza los sitios y redes de dos diarios impresos, Clarín y La Nación: sería interesante incorporar más sitios de distintos medios y de diversos países. Segundo, la investigación examina la producción y difusión de noticias, de manera que se deja afuera la agenda de los usuarios. Una investigación a futuro podría estudiar qué noticias consumen los usuarios, con cuáles se involucran, tanto marcándolas como favoritas como contestando como compartiéndolas, y si coinciden con las propuestas por los editores. Finalmente, la divergencia en espacios de comunicación del mismo medio sugiere que el po- 
der de fijación de la agenda tanto intramedia como inter-media parece estar en retirada, aunque habría que comparar las agendas de las redes con los temas considerados más importantes por los votantes para identificar la influencia de las distintas agendas.

\section{Conclusiones}

Esta investigación encuentra que los dos principales diarios en papel de la Argentina, Clarín y La Nación, mantienen una agenda relacionada a los asuntos públicos en sus páginas de inicio, mientras que en las redes sociales priorizan noticias vinculadas a los asuntos no públicos, de manera más marcada en Facebook que en Twitter. Por otra parte, el análisis de los sitios y las cuentas en Facebook y Twitter en distintos momentos del día muestra que la divergencia es menor temprano por la mañana y aumenta a lo largo del día. A partir de estos hallazgos este trabajo indaga los motivos de esta selección y las diferentes temáticas que seleccionan los medios.

Estos hallazgos tienen diversas implicancias para el estudio de los medios y el ciclo de noticias, las redes sociales, y los procesos de fijación de agenda en la sociedad contemporánea. En primer lugar, indican que la lógica de las redes sociales incide en la difusión de noticias por parte de actores establecidos de la industria. Los usuarios de redes tienden a estar menos interesados en noticias de asuntos públicos que los lectores tradicionales de diarios, y esa agenda de entretenimiento termina reflejada en las cuentas que los mismos periódicos manejan. Las audiencias les dicen a los medios sobre qué quieren pensar, a través del monitoreo de sus preferencias en los sitios y en las redes sociales, y las cuentas de los medios de noticias tienden a reflejar esos intereses.

Segundo, los hallazgos presentados por esta investigación indican que las redes sociales difieren entre sí, ya que tanto Clarín como La Nación postean menos noticias sobre política, economía y asuntos internacionales en Facebook que en Twitter. Cada red social tiene sus propias características, basadas en las prácticas de los usuarios (Boczkowski, Matassi y Mitchelstein, 2018; Kümpel, Karnowski y Keyling, 2015) y los medios de noticias se adaptan a esos usos diversos, proponiendo agendas distintas para cada espacio virtual.

Por último, la persistencia de los asuntos de temas públicos tanto en los sitios como en las redes en las primeras horas de la mañana, sugiere que, más allá de las crisis en los periódicos impresos, los diarios mantienen relevancia a la hora de fijar agenda al principio de la jornada. La discusión en redes sociales sobre las portadas de los diarios en redes sociales podría asemejarse a la práctica por la cual las radios empiezan sus programas de noticias matutinos leyendo las noticias de tapa. A lo largo del día, conforme aparecen noticias tanto en medios tradicionales como en redes, esa primera agenda del diario impreso se diluye. El ciclo de noticias no colapsa, sino que se transforma. La política no desaparece completamente de las redes, si no que abandona con el transcurso de las horas el primer plano, para volver a aparecer al día siguiente, como el diario en papel. 
En conjunto, estos hallazgos indican que las particularidades de cada medio, cada red social y cada momento del día deben ser tenidas en cuenta a la hora de analizar procesos de fijación de agenda en internet en general y en redes sociales en particular. Esperamos que esta investigación sea un paso fructífero en esa dirección.

\section{Bibliografía}

Althaus, S. L. y Tewksbury, D. (2002). Agenda setting and the "new" news: Patterns of issue importance among readers of the paper and online versions of the New York Times. Communication Research, 29(2), 180-207.

Aruguete, N. (2009). Estableciendo la agenda. Los orígenes y la evolución de la teoría de la Agenda Setting. Ecos de la comunicación, (2).

Aruguete, N. (2015). El poder de la agenda: política, medios y público. Editorial Biblos.

Bandari, R., Asur, S. y Huberman, B. A. (2012). The pulse of news in social media: Forecasting popularity. ICWSM, 12, 26-33.

Boczkowski, P. J. y De Santos, M. (2007). When more media equals less news: Patterns of content homogenization in Argentina's leading print and online newspapers. Political Communication, 24(2), 167-180.

Boczkowski, P. J., Matassi, M. y Mitchelstein, E. (2018). How Young Users Deal With Multiple Platforms: The Role of Meaning-Making in Social Media Repertoires. Journal of ComputerMediated Communication, 23(5), 245-259.

Boczkowski, P. J., Mitchelstein, E. y Matassi, M. (2018). "News comes across when I'm in a moment of leisure": Understanding the practices of incidental news consumption on social media. New Media \& Society.

Boyd, D., Golder, S. y Lotan, G. (2010, January). Tweet, tweet, retweet: Conversational aspects of retweeting on twitter. In System sciences (HICSS), $201043 \mathrm{rd} \mathrm{Hawaii} \mathrm{international}$ conference on (pp. 1-10). IEEE.

Canavilhas, J. (2007). Web journalism: from the inverted pyramid to the tumbled pyramid. Covilhā, Portugal: Biblioteca On-line deficiencias da comunicaçao.

Campos Freire, F. (2008). Las redes sociales trastocan los modelos de los medios de comunicación tradicionales. Revista Latina de comunicación social, 11(63).

Castillo, C., El-Haddad, M., Pfeffer, J.y Stempeck, M. (2014, February). Characterizing the life cycle of online news stories using social media reactions. In Proceedings of the 17th ACM conference on Computer supported cooperative work \& social computing (pp. 211-223). ACM.

Chaffee, S. H. y Metzger, M. J. (2001). The end of mass communication? Mass communication \& society, 4(4), 365-379.

Chaykowsky, K. (2018). Facebook Focuses News Feed On Friends And Family, Curbing The Reach Of Brands And Media. Forbes. Recuperado de: https://www.forbes.com/sites/kathleenchaykowski/2018/01/11/

facebook-focuses-news-feed-on-friends-and-family-curbing-the-reach-of-brands-and-media.

Cohen, B. C. (1963). The press and foreign policy. Princeton, NJ: Princeton University Press. 
Conway, B. A., Kenski, K. y Wang, D. (2015). The rise of Twitter in the political campaign: Searching for intermedia agenda-setting effects in the presidential primary. Journal of Computer-Mediated Communication, 20 (4), 363-380.

Dearing, J. W. y Rogers, E. (1996). Agenda-setting. Sage publications.

Donsbach, W. (2004). Psychology of news decisions: Factors behind journalists' professional behavior. Journalism, 5(2), 131-157.

Fletcher, R. y Nielsen, R. K. (2017). Are news audiences increasingly fragmented? a crossnational comparative analysis of cross-platform news audience fragmentation and duplication. Journal of Communication, 67(4), 476-498.

García de Torres, E., Yezers'ka, L., Rost, A., Calderín, M., Edo, C., Rojano, M. y Corredoira, L. (2011). Uso de Twitter y Facebook por los medios iberoamericanos. El profesional de la información, 20(6), 611-620.

García, R. (2014). Twitter y la teoría de la Agenda-Setting: mensajes de la opinión pública digital. Estudios sobre el mensaje periodístico, 20(1), 249-265.

González Molina, S. y Ortells Badenes, S. (2012). La polivalencia periodística de los profesionales en las redes sociales. Estudios sobre el Mensaje Periodístico. Vol. 18 Núm. especial octubre, 455463.

González Molina, S. y Ramos del Cano, F. (2013). El uso periodístico de Facebook y Twitter: un análisis comparativo de la experiencia europea. Historia y Comunicación Social, 18, 419-433.

Harder, R. A., Sevenans, J. y Van Aelst, P. (2017). Intermedia agenda setting in the social media age: How traditional players dominate the news agenda in election times. The International Journal of Press/Politics, 22(3), 275-293.

Hille, S.y Bakker, P. (2013). I like news. Searching for the 'Holy Grail'of social media: The use of Facebook by Dutch news media and their audiences. European Journal of Communication, 28(6), 663-680.

Hong, S. (2012). Online news on Twitter: Newspapers' social media adoption and their online readership. Information Economics and Policy, 24(1), 69-74.

Jacobson, S. (2013). Does audience participation on Facebook influence the news agenda? A case study of the Rachel Maddow Show. Journal of Broadcasting E Electronic Media, 57(3), 338-355.

Jerónimo, P. y Duarte, Â. (2010). Twitter e jornalismo de proximidade: estudo de rotinas de produção nos principais títulos de imprensa regional em portugal. Prisma. com, (12), 114-135.

Johnson, T. y Perlmutter, D. (2009). "The Facebook Election: New Media and the 2008 Election Campaign” Special Symposium.

Jung, J. Y. y Villi, M. (2018). Newspapers and Cross-Level Communications on Social Media: A comparative study of Japan, Korea, and Finland. Digital Journalism, 6(1), 58-75.

Kalsnes, B. (2016). Intermedia Agenda Setting: Political Journalists' Source Hunting on Social Media. En Jensen, J. L., Mortensen, M., y Ørmen, J (eds.) News Across Media (pp. 22-41). Routledge. 
Klinenberg, E. (2005). Convergence: News production in a digital age. The Annals of the American Academy of Political and Social Science, 597(1), 48-64.

Kosicki, G. M. (1993). Problems and opportunities in agenda-setting research. Journal of communication, 43(2), 100-127.

Krane, M. (2010). The socially filtered media agenda: a study of agenda setting among news outlets on Twitter (Tesis de doctorado, University of Missouri--Columbia).

Krippendorf, K, (2018). Content analysis: An introduction to its methodology. Thousand Oaks, CA: Sage.

Krippendorf, K y Bock, M.A., (2018). The content analysis reader. Thousand Oaks, CA: Sage.

Krippendorff, K. y Bock, M. A. (2009). The content analysis reader. Sage.

Kümpel, A. S., Karnowski, V. y Keyling, T. (2015). News sharing in social media: A review of current research on news sharing users, content, and networks. Social media + society, 1(2), 2056305115610141.

Kwak, H., Lee, C., Park, H. y Moon, S. (2010, April). What is Twitter, a social network or a news media? In Proceedings of the 19th international conference on World wide web (pp. 591600). AcM.

Lara, T. (2008). La nueva esfera pública. Los medios de comunicación como redes sociales. Telos, 76, 128-131.

Leskovec, J., Backstrom, L. y Kleinberg, J. (2009, June). Meme-tracking and the dynamics of the news cycle. In Proceedings of the 15th ACM SIGKDD international conference on Knowledge discovery and data mining (pp. 497-506). ACM.

Lasorsa, D. L., Lewis, S. C. y Holton, A. E. (2012). Normalizing Twitter: Journalism practice in an emerging communication space. Journalism studies, 13(1), 19-36.

López-Escobar, E., Llamas, J. P. y McCombs, M. (1996). Una dimensión social de los efectos de los medios de difusión: agenda-setting y consenso. Comunicación y Sociedad, 9 (1 y 2), 91-125.

Martin, S. y Grüb, B. (2016). Towards a process of agenda setting driven by social media. International Journal of Energy Sector Management, 10(1), 38-55.

McCombs, M. (2005). A look at agenda-setting: Past, present and future. Journalism studies, 6(4), 543-557.

McCombs, M. E. y Shaw, D. L. (1972). The agenda-setting function of mass media. Public opinion quarterly, 36(2), 176-187.

McCombs, M. y Evatt, D. (1995). Issues and attributes: Exploring a new dimension in agenda setting. Communicacion y Sociedad, 8(1), 7-32.

Meraz, S. (2009). Is there an elite hold? Traditional media to social media agenda setting influence in blog networks. Journal of Computer-Mediated Communication, 14(3), 682-707.

Meraz, S. (2011). Using time series analysis to measure intermedia agenda-setting influence in traditional media and political blog networks. Journalism \& Mass Communication Quarterly, 88(1), 176-194.

Messner, M. y Distaso, M. W. (2008). The source cycle: How traditional media and weblogs use each other as sources. Journalism Studies, 9(3), 447-463. 
Messner, M. y Garrison, B. (2011). Study shows some blogs affect traditional news media agendas. Newspaper Research Journal, 32(3), 112-126.

Mitchelstein, E. y Boczkowski, P. J. (2018). Juventud, estatus y conexiones. Explicación del consumo incidental de noticias en redes sociales. Revista mexicana de opinión pública, (24), 131-145.

Newman, N., Fletcher, R., Kalogeropoulos, A., Levy, D. A. y Nielsen, R. K. (2017). Reuters Institute digital news report 2017.

Noguera Vivo, J. M. (2010). Redes sociales como paradigma periodístico. Medios españoles en Facebook. Revista Latina de Comunicación Social, 75 (175-186).

Oeldorf-Hirsch, A., Birnholtz, J.y Hancock, J. T. (2017). Your post is embarrassing me: Face threats, identity, and the audience on Facebook. Computers in human behavior, 73, 92-99.

Parmelee, J. H. (2014). The agenda-building function of political tweets. New Media \& Society, 16(3), 434-450.

Raimondo Anselmino, N. y Bertone, M. (2013). Prensa y Redes Sociales en Internet: Aproximaciones a la relación de dos diarios argentinos en línea con Facebook y Twitter.

Roberts, M., Wanta, W. y Dzwo, T. H. (2002). Agenda setting and issue salience online. Communication Research, 29(4), 452-465.

Rodríguez Díaz, R. (2004). Teoría de la Agenda-Setting: aplicación a la enseñanza universitaria. Observatorio Europeo de Tendencias Sociales.

Rogers, E., \& Dearing, J. (1988). Agenda-setting research: Where has it been, where is it In JA Anderson (Ed.), Communication yearbook 11 (pp. 555-594).

Rost, A. (2012). Modelos de uso y gestión de redes sociales en el Periodismo. In Trabajo presentado en el IV Congreso Internacional de Ciberperiodismo y Web (Vol. 2).

Sayre, B., Bode, L., Shah, D., Wilcox, D. y Shah, C. (2010). Agenda setting in a digital age: Tracking attention to California Proposition 8 in social media, online news and conventional news. Policy \& Internet, 2(2), 7-32.

Semetko, H. A. y Canel, M. J. (1997). Agenda-senders versus agenda-setters: Television in Spain's 1996 election campaign. Political Communication, 14(4), 459-479.

Skogerbø, E. y Krumsvik, A. H. (2015). Newspapers, Facebook and Twitter: Intermedial agenda setting in local election campaigns. Journalism Practice, 9(3), 350-366.

Sweetser, K. D., Golan, G. J. y Wanta, W. (2008). Intermedia agenda setting in television, advertising, and blogs during the 2004 election. Mass Communication \& Society, 11(2), 197-216.

Valeriani, A. y Vaccari, C. (2016). Accidental exposure to politics on social media as online participation equalizer in Germany, Italy, and the United Kingdom. New Media \& Society, $18(9), 1857-1874$.

Vis, F. (2013). Twitter as a reporting tool for breaking news: Journalists tweeting the 2011 UK riots. Digital journalism, 1(1), 27-47.

Weaver, D. y Elliott, S. N. (1985). Who sets the agenda for the media? A study of local agendabuilding. Journalism Quarterly, 62(1), 87-94.

Wright, D. K. y Hinson, M. D. (2008). How blogs and social media are changing public relations and the way it is practiced. Public relations journal, 2(2), 1-21. 